\title{
Prospective Survey of Chest X-Rays or Computed Tomography Abnormalities in Patients with Inflammatory Bowel Disease Treated with Infliximab
}

Hiroyuki Miyazaki ${ }^{1 *}$, Nobuhiko Nagata1, Takanori Akagi ${ }^{1}$, Masaru Kodama1, Makoto Tanaka1, Satoshi Takeda1, Taishi Harada1, Toshiyuki Matsui $^{2}$, Masaki Fujita ${ }^{3}$ and Kentaro Watanabe ${ }^{3}$

${ }^{1}$ Department of Respiratory Medicine, Fukuoka University, Chikushi Hospital, Fukuoka, Japan

${ }^{2}$ Department of Gastroenterology, Fukuoka University, Chikushi Hospital, Fukuoka, Japan

${ }^{3}$ Department of Respiratory Medicine, Faculty of Medicine, Fukuoka University, Fukuoka, Japan

\begin{abstract}
Background: Patients with inflammatory bowel disease (IBD) occasionally present with pulmonary involvement. Recently, a tumor necrosis factor- $\alpha$ (TNF- $\alpha$ ) blocking agent was introduced for the treatment of IBD in Japan. We aimed to clarify the frequency and type of chest X-rays or computed tomography (CT) abnormalities associated with IBD in patients treated with infliximab (IFX).

Methods: We prospectively obtained, through interviews, information regarding respiratory symptoms, medical history, smoking history, occupational and environmental exposure, and medication history for patients with IBD treated with IFX at the IBD center of our hospital. We also examined percutaneous oxygen saturation $\left(\mathrm{SpO}_{2}\right)$ and chest $\mathrm{X}$-rays. Chest computed tomography and spirometry were also performed for patients with respiratory symptoms, abnormal $\mathrm{SpO}_{2}$, and/or abnormal chest X-rays.

Results: Subjects were 115 patients with Crohn's disease (CD) and 8 with ulcerative colitis (UC). Of those with CD, 21 presented with respiratory symptoms. Among these, 4 (3.5\%) had chest X-ray abnormalities. None of the patients with UC had respiratory symptoms or chest X-ray abnormalities. CT examination of 13 patients with CD revealed 5 with bronchiectatic changes, ground glass opacity (GGO), consolidation, nodular lesions, and/or granular shadows.
\end{abstract}

Conclusion: Chest X-rays or computed tomography abnormalities were detected in at least $3.5 \%$ of patients with CD treated with IFX.

Keywords: Pulmonary lesions; Inflammatory bowel disease; Infliximab; Crohn's disease; Ulcerative colitis

\section{Introduction}

A number of studies have reported on respiratory disorders associated with inflammatory bowel disease (IBD) [1-9]. For instance, Kraft et al. [1] reported that the frequency of pulmonary lesions associated with IBD was $0.21 \%$. Camus et al. [2] reported that patterns of involvement included airway inflammation, in the form of subglottic stenosis, chronic bronchitis, severe chronic bronchial suppuration, bronchiectasis, and chronic bronchiolitis. Marvisi and Fornasari [3] reported that the most frequent manifestation is bronchial inflammation and suppuration with or without bronchiectasis. Some patients present severe tracheal inflammation and obstruction with an inflammatory mass bulging into the tracheal lumen. Lung parenchymal disorder is relatively uncommon. BOOP is the most commonly reported parenchymal manifestation of IBD [4,5]. Other studies reported the incidence of thrombolic events in IBD patients is three to four times higher than in age-matched control subjects $[4,6]$.

In another study [7], respiratory disorders in patients with IBD were frequently induced by sulfasalazine or mesalamine, which leads to eosinophilic pneumonia and fibrosing alveolitis, or by treatment with methotrexate, which can lead to pneumonitis. Yilmaz et al. [8] examined lung function in patients with IBD and found abnormalities in 22 of the 39 patients examined. Among these patients, the most prevalent abnormalities were decreased forced expiratory volume in $1 \mathrm{~s}$ $\left(\mathrm{FEV}_{1}\right), \mathrm{FEV}_{1}$ / forced vital capacity (FVC), forced expiratory flow (FEF) $25 \%$ to $75 \%$, transfer coefficient for carbon monoxide (DLCO), and DLCO/alveolar volume. One study found that increased respiratory symptoms were associated with a high endoscopic activity index in patients with ulcerative colitis (UC) [8]. It has also been reported that a very broad spectrum of respiratory disorders occurs in patients with IBD. Diseases of the large airways are the most common form of involvement, with bronchiectasis being the most frequently reported form of IBD-associated lung disease [9]. Recently, anti-tumor necrosis factor (TNF- $\alpha$ ) drugs have been used to treat IBD. However, little is known about pulmonary lesions associated with IBD in patients treated with these agents in Japan. To this end, the present study aimed to clarify the frequency and type of chest X-rays or computed tomography abnormalities associated with IBD in patients treated with infliximab (IFX) at the IBD center of Fukuoka University, Chikushi Hospital, Chikushino City, Fukuoka, Japan.

\section{Methods}

We prospectively interviewed patients with IBD who were treated with IFX at the IBD center of our hospital and agreed to participate in the present study. The study duration was from October 2011 to March 2012.

Through the interviews, we obtained information regarding: 1) respiratory symptoms, 2) past history of respiratory diseases, 3)

${ }^{*}$ Corresponding author: Hiroyuki Miyazaki, Department of Respiratory Medicine Fukuoka University, Chikushi Hospital, 1-1-1 Zokumyoin, Chikushino-City, Fukuoka-818-8502, Japan, Tel: +81929211011; Fax: +81929283890; E-mail: miyahiro@fukuoka-u.ac.jp

Received March 26, 2016; Accepted October 28, 2016; Published October 31 2016

Citation: Miyazaki H, Nagata N, Akagi T, Kodama M, Tanaka M, et al. (2016) Prospective Survey of Chest X-Rays or Computed Tomography Abnormalities in Patients with Inflammatory Bowel Disease Treated with Infliximab. J Pulm Respir Med 6: 378. doi: 10.4172/2161-105X.1000378

Copyright: ( 2016 Miyazaki H, et al. This is an open-access article distributed under the terms of the Creative Commons Attribution License, which permits unrestricted use, distribution, and reproduction in any medium, provided the original author and source are credited. 
Citation: Miyazaki H, Nagata N, Akagi T, Kodama M, Tanaka M, et al. (2016) Prospective Survey of Chest X-Rays or Computed Tomography Abnormalities in Patients with Inflammatory Bowel Disease Treated with Infliximab. J Pulm Respir Med 6: 378. doi: 10.4172/2161105X.1000378

Page 2 of 4

comorbid respiratory diseases, 4) smoking history, 5) employment status, 6) residential status, 7) presence of pets, 8) having contact or not with birds, 9) feather product use, and 10) daily medication. We also assessed percutaneous oxygen saturation $\left(\mathrm{SpO}_{2}\right)$ and chest $\mathrm{X}$-rays for all patients. Chest X-rays were performed by Aero-DR (Konica Minolta, Japan). Chest computed tomography (CT) and spirometry were performed for patients with respiratory symptoms, chest X-rays abnormalities, and/or decreased $\mathrm{SpO}_{2}(<95 \%)$. Chest CTs were performed by Definition AS (64 slices) or Sensation cardiac 64 (64 slices) (Siemens, Germany). The sectioning thickening was $5 \mathrm{~mm}$, respectively.

Chest X-rays and CT examinations were independently interpreted by three doctors of respiratory medicine. This study was approved by the Institutional Review Board of our hospital and informed consent was obtained from all subjects.

\section{Results}

Subjects were 115 and 8 patients with Crohn's disease (CD) and UC, respectively. Their clinical features are summarized in Table 1 . Patients with CD were younger than those with UC. Because IFX was recently approved for use to treat UC in Japan, the number of patients with UC in this study was limited.

Median disease durations for CD and UC were 7.4 years and 4.3 years, respectively. The number of patients with $\mathrm{CD}$ who were current smokers, ex-smokers, or non-smokers was 40,22 , and 53, respectively, and 1,3 , and 4 for those with UC. Mesalazine was administered to 82 patients with $\mathrm{CD}$ and 4 patients with UC, and oral steroids were administered to 78 patients with CD and 4 patients with UC.

The number of patients with respiratory symptoms such as cough, sputum, or dyspnea, chest X-rays abnormalities, and reduced $\mathrm{SpO}_{2}$ is shown in Table 2. Respiratory symptoms were found in 21 of 115 patients with CD (cough, 9; sputum, 3; dyspnea, 3; cough and sputum, 4 ; cough, sputum and dyspnea, 6 ). The symptom of one case associated with cough disappeared spontaneously during the observation period. On the other hand, the symptoms of other cases remained unchanged. None of the patients with UC had respiratory symptoms. Chest X-ray abnormalities were observed in 4 of 115 patients with $\mathrm{CD}$, but in none of the patients with UC. All 4 of these patients presented with respiratory symptoms. $\mathrm{SpO}_{2}$ was in the normal range for all patients with $\mathrm{CD}$ and UC. The chest X-ray abnormal findings of these four cases did not change during the observation period at least 2 years.

Eight of 21 patients did not visit our hospital again; CT examination and spirometry were performed for 13 patients with chest X-ray abnormalities $(n=4)$ and/or respiratory symptoms $(n=13)$. None of these 13 patients had no abnormal findings, whereas four had

\begin{tabular}{|l|c|c|}
\hline & CD & UC \\
\hline Patients (n) & 115 & 8 \\
\hline Sex (M/F) & $87 / 28$ & $5 / 3$ \\
\hline Age (years) & $38.0(26-60)$ & $50.0(24-58)$ \\
\hline Disease duration (years) & $7.4(0.25-21.0)$ & $4.3(0.75-10.5)$ \\
\hline $\begin{array}{l}\text { Smoking history (smoker lex-smoker/non- } \\
\text { smoker) }\end{array}$ & $40 / 22 / 53$ & $1 / 3 / 4$ \\
\hline Period of IFX therapy (years) & $3.8(0.4-7.6)$ & $2.2(0.4-3.4)$ \\
\hline Treated with mesalazine $(n)$ & 82 & 4 \\
\hline Treated with oral steroids $(n)$ & 78 & 4 \\
\hline
\end{tabular}

CD: Crohn's Disease; UC: Ulcerative Colitis; IFX: Infliximab; Data are presented as number of patients or median (range)

Table 1: Patient characteristics.

\begin{tabular}{|c|c|c|}
\hline & CD (n=115) & UC (n=8) \\
\hline Respiratory symptoms & 21 & \multirow{2}{*}{0} \\
\hline Cough & 15 & \\
\hline Sputum & 9 & \\
\hline Dyspnea & 5 & \\
\hline Chest X-ray abnormalities & 4 & \multirow{2}{*}{0} \\
\hline GGO & 2 & \\
\hline Consolidation & 1 & \\
\hline Linear shadow & 1 & 0 \\
\hline Nodular shadow & 1 & 0 \\
\hline Decreased $\mathrm{SpO}_{2}(<95 \%)$ & 0 & \\
\hline
\end{tabular}

CD: Crohn's Disease; UC: Ulcerative Colitis; GGO: Ground Glass Opacity; $\mathrm{SpO}_{2}$ Percutaneous Oxygen Saturation; Data are presented as number of patients.

Table 2: Respiratory symptoms, abnormalities on chest X-rays, and $\mathrm{SpO}_{2}$ status.

abnormal findings, including bronchiectatic changes, ground glass opacity (GGO), consolidation, nodular lesions, and granular shadow in $2,1,1,2$, and 1 patients, respectively (Table 3 ). Two patients had more than one abnormal finding, including one with bronchiectatic changes and nodular shadow, and the other with bronchiectatic changes, consolidation, and granular shadow.

Figure 1 shows a nodular and bronchiectatic shadows in the lung of a 62-year-old male and chest CT taken 23 months later revealed no significant change of these shadows. Figure 2 shows GGO in the lung of a 27-year-old male. Figure 3 shows a granular, bronchiectatic shadow with consolidation in a 58-year-old female and chest CT taken 38 months later revealed progression of bronchiectatic change and increase of granular shadow. Figure 4 shows a nodular shadow in the lung of a 62-year-old male and chest CT taken 28 months later revealed no significant change of the shadow.

Spirometry results are summarized in Table 4. All patients had normal values.

\section{Discussion}

Respiratory complications have been reported in some patients with IBD [1-4], and include diseases of large airways, bronchiectasis, drug-induced pneumonitis, and lung function abnormalities (e.g., obstructive changes or diffusion disturbances). Recently, anti-TNF inhibitors have been used to treat IBD. These inhibitors are known to induce pulmonary complications such as tuberculosis (TB) and interstitial lung disease (ILD). For patients with IBD, the incidence of active TB after treatment with an anti-TNF inhibitor was reported to be approximately $1 \%$ to $2 \%$ [10], whereas the incidence of ILD associated with these inhibitors is unknown. However, there are reported instances of ILD associated with IBD during treatment with anti-TNF inhibitors [11].

To our knowledge, this is the first prospective study on pulmonary lesions found in patients with IBD treated with IFX in Japan.

Radiographic abnormalities are often seen with high resolution CT in patients with IBD. For example, in one study, 25 of 39 patients with IBD presented with radiographic abnormalities with this modality $[12,13]$. Although our study was limited to assessing patients with respiratory symptoms and/or chest X-ray abnormalities by CT, 4 of 13 patients were found to have abnormalities (30.8\%). This discrepancy between studies might be explained by differences in subject characteristics, or the fact that only those treated with IFX were included in this study.

With respect to abnormalities on CT images, they could be 1) a result of pulmonary lesions associated with IBD itself, 2) drug-induced, 3) infection-related, or 4) related to conditions other than IBD, 
Citation: Miyazaki H, Nagata N, Akagi T, Kodama M, Tanaka M, et al. (2016) Prospective Survey of Chest X-Rays or Computed Tomography Abnormalities in Patients with Inflammatory Bowel Disease Treated with Infliximab. J Pulm Respir Med 6: 378. doi: 10.4172/2161105X.1000378

\begin{tabular}{|c|c|}
\hline CT findings & Number \\
\hline None & 9 \\
\hline Bronchiectatic changes & 2 \\
\hline Ground glass opacity & 1 \\
\hline Consolidation & 1 \\
\hline Nodular lesion & 2 \\
\hline Granular shadow & 1 \\
\hline
\end{tabular}

Table 3: CT findings in patients with chest X-ray abnormalities $(n=13)$. Two patients presented with more than two findings (one with bronchiectatic changes and nodular shadow, and the other with bronchiectatic changes, consolidation, and granular shadow). Data are presented as number of patients.

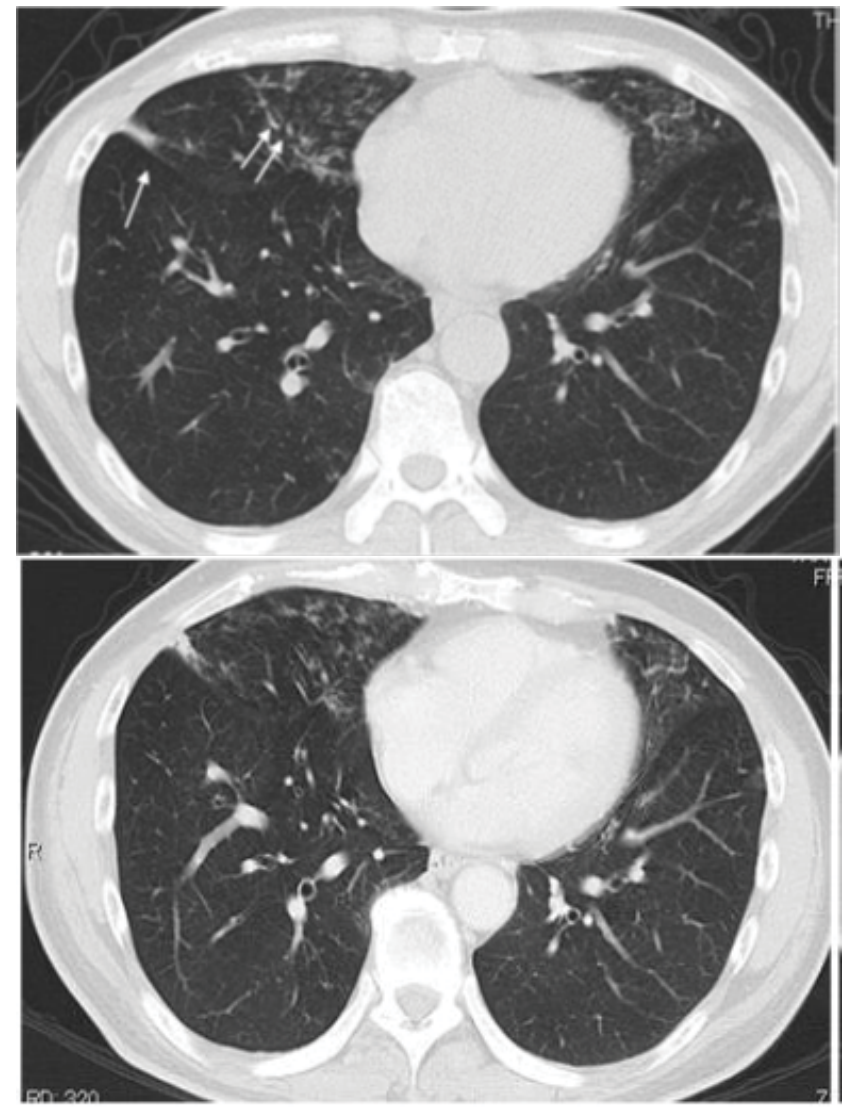

Figure 1: 62-year-old male. Computed tomography taken at the entry to the study shows a nodular shadow (arrow) and bronchiectasis (double arrows) (top). These shadows did not change 23 months later (bottom).

infection, or drugs. The pulmonary lesions noted in this study were different from those reported as being drug-induced in other studies $[11,13]$, and did not change even with continued IFX or mesalazine administration in two cases. Bronchiectatic and granular shadows noted in one case increased during 38-months period. Blood and sputum tests for mycobacterial and fungal infections came back negative in this case. Moreover, abnormal shadows noted on CT images were consistent with those reported as IBD associated lesions. Therefore, we considered that these shadows were possibly associated with IBD itself. Thickening of the bronchial wall and ground glass opacities are also often observed in patients with IBD [12]. In one study [13], bronchiectasis was observed in 13 of 17 patients assessed. Although the number of patients assessed by CT was smaller in the present study, the findings were similar to those reported elsewhere. Finally, sarcoidosis shares many common characteristics with IBD, especially CD, as they both are granulomatous diseases [14]. Hilar or mediastinal lymph node swelling is not found

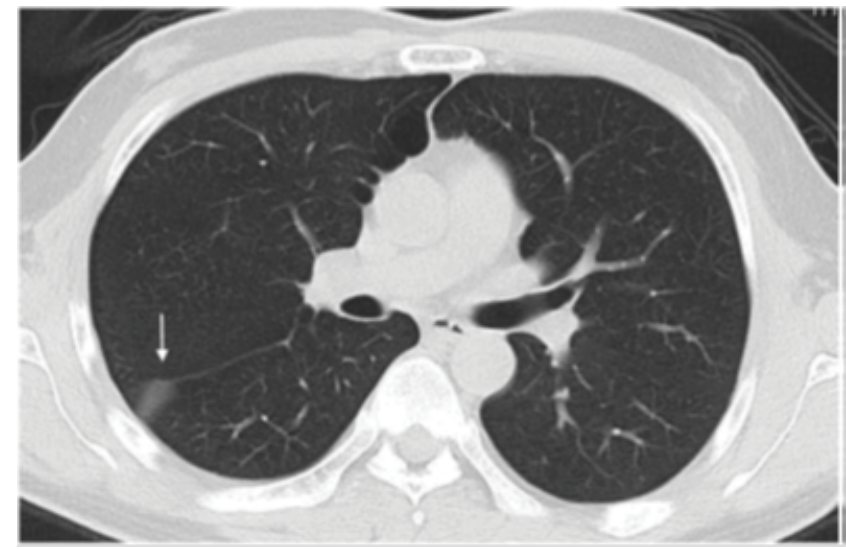

Figure 2: 27-year-old male. Computed tomography shows a ground glass opacity (arrow).

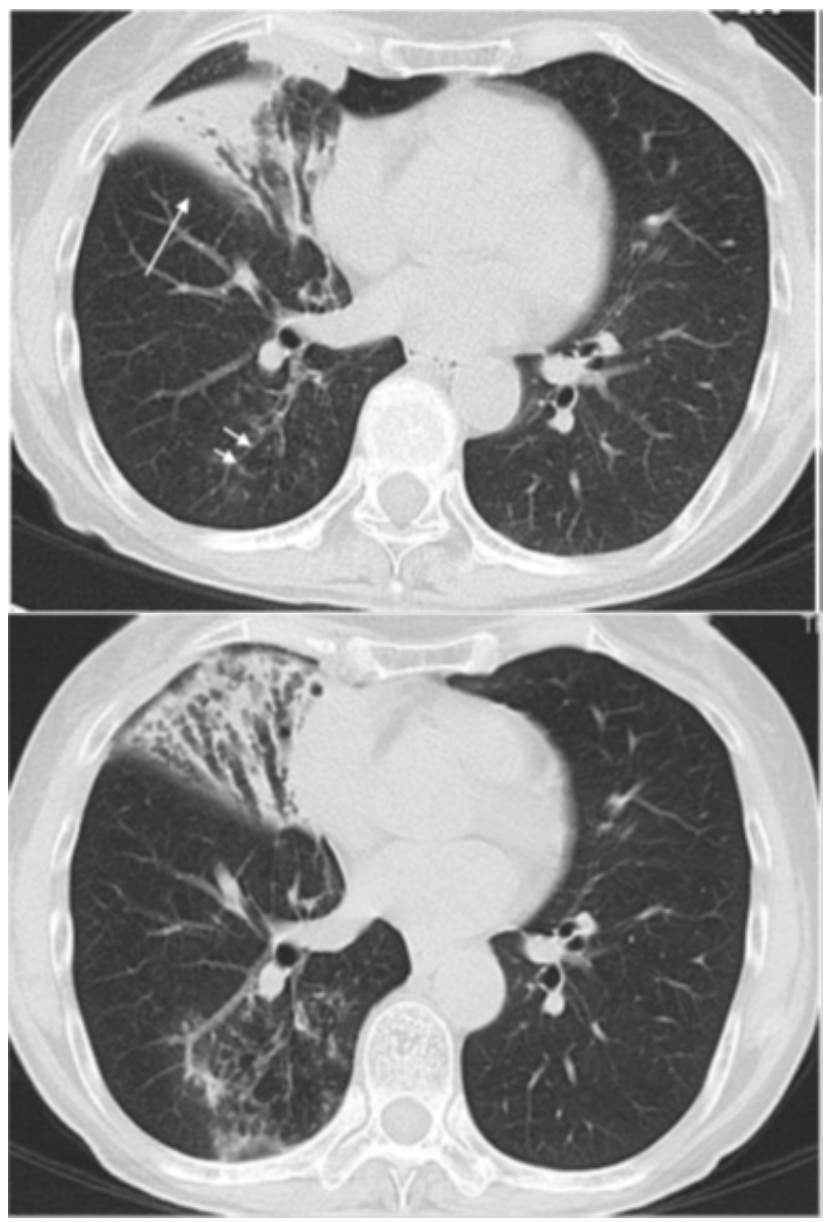

Figure 3: 58-year-old female. Computed tomography taken at the entry to the study shows a bronchiectatic shadow with consolidation (arrow) and granular shadow (double arrows) (top). Chest CT taken 38 months later revealed progression of bronchiectatic change and increase of granular shadow (bottom)

in any CT images of our cases, though the possibility of sarcoidosis cannot be denied. We cannot rule out the possibility that the lesions were incidental findings.

Previous studies have also demonstrated obstructive changes and 
Citation: Miyazaki H, Nagata N, Akagi T, Kodama M, Tanaka M, et al. (2016) Prospective Survey of Chest X-Rays or Computed Tomography Abnormalities in Patients with Inflammatory Bowel Disease Treated with Infliximab. J Pulm Respir Med 6: 378. doi: 10.4172/2161105X.1000378

Page 4 of 4

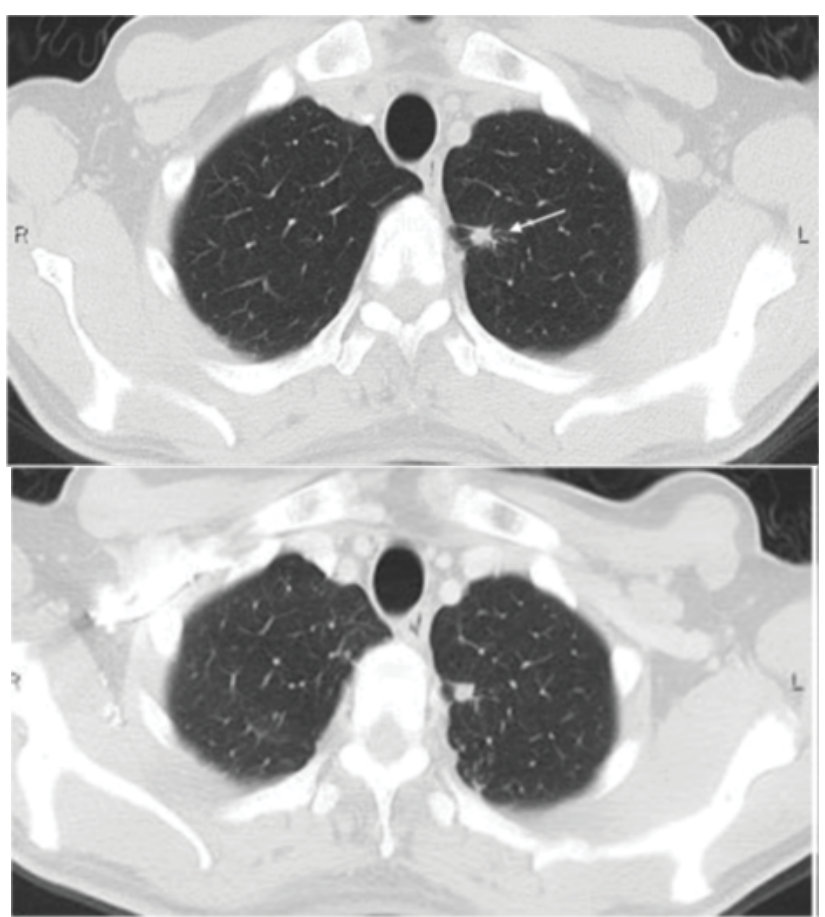

Figure 4: 62-year-old male. Computed tomography shows a nodular shadow (arrow) (top). The nodule did not change 28 month later (bottom).

\begin{tabular}{|c|c|}
\hline Items of spirometry & Results \\
\hline $\mathrm{VC}(\mathrm{L})$ & $4.20(3.41-4.85)$ \\
\hline$\% \mathrm{VC}(\%)$ & $104.5(85.8-133.1)$ \\
\hline $\mathrm{FVC}(\mathrm{L})$ & $4.16(3.37-5.82)$ \\
\hline $\mathrm{FEV}_{1.0}(\mathrm{~L})$ & $3.58(2.76-4.85)$ \\
\hline $\mathrm{FEV}_{1.0} / \mathrm{FVC}(\%)$ & $85.73(77.57-95.21)$ \\
\hline
\end{tabular}

VC: Vital Capacity, \%VC: Percent Vital Capacity, FVC: Forced Vital Capacity; $\mathrm{FEV}_{1.0}$ : Forced Expiratory Volume $1.0(\mathrm{Sec})$; Data are presented as median (range).

Table 4: Spirometry for patients presenting with chest X-ray abnormalities $(n=13)$.

diffusion disturbances by spirometory [15-17]. Obstructive changes were not observed in all cases in the present study, but the number of patients who underwent spirometry was limited. Diffusion capacity could not be compared with that of other studies, as it was not assessed in this study.

Sixteen patients (13.9\%) who presented with respiratory symptoms, but without chest X-ray abnormalities, had normal spirometry results. All of these patients had a history of smoking (average of 15.2 packs/ year). Respiratory symptoms in these patients were considered to be related to smoking, rather than to IBD itself, although spirometry did not reveal any abnormalities such as obstructive disturbances.

This study has some limitations. First, only patients with IBD who were administered IFX were assessed. Since IFX was only recently approved for use in treating UC in Japan, the number of patients with UC was limited. Second, CT was performed only for patients who presented with respiratory symptoms and/or chest X-ray abnormalities. Thirdly, the histological examination or broncho-alveolar lavage
(BAL) was not done in any patients whose chest X-ray or CT revealed abnormal shadows, therefore, we could not show the true nature of the shadows.

In conclusion, pulmonary lesions observed in patients with $\mathrm{CD}$ who were treated with IFX were present in at least $3.5 \%$ of these patients.

\section{References}

1. Kraft SC, Earle RH, Roesler M, Esterly JR (1976) Unexplained bronchopulmonary disease with inflammatory bowel disease. Arch Intern Med 136: 454-459

2. Camus P, Piard F, Ashcroft T, Gal AA, Colby TV (1993) The lung in inflammatory bowel disease. Medicine (Baltimore) 72: 151-183.

3. Marvisi M, Fornasari G (2001) Is the lung a target organ in inflammatory bowel disease? Recent Prog Med 92: 774-777.

4. Ji XQ, Wang LX, Lu DG (2014) Pulmonary manifestation of inflammatory bowel disease. World J Gastroenterol 20: 13501-13511.

5. Gil-Simón P, Barrio Andrés J, Atienza Sánchez R, Julián Gómez L, López Represa C, et al. (2008) Bronchiolitis obliterans organizing pneumonia and Crohn's disease. Rev Esp Enferm Dig 100: 175-177.

6. Miehsler W, Reinisch W, Valic E, Osterode W, Tillinger W, et al. (2004) Is inflammatory bowel disease an independent and disease specific risk factor for thromboembolism? Gut 53: 542-548.

7. Schleiermacher D, Hoffmann JC (2007) Pulmonary abnormalities in inflammatory bowel disease. J Crohns Colitis 1: 61-69.

8. Yilmaz A, Demirci NY, Hosgun D, Uner E, Erdogan Y, et al. (2010) Pulmonary involvement in inflammatory bowel disease. World J Gastroenterol 16: 4952-4957.

9. Black H, Mendoza M, Murin S (2007) Thoracic manifestations of inflammatory bowel disease. Chest 131: 524-532.

10. Kim ES, Song GA, Cho KB, Park KS, Kim OK, et al. (2015) Significant risk and associated factors of active tuberculosis infection in Korean patients with inflammatory bowel disease using anti-TNF agents. World J Gastroenterol 21 : 3308-3316.

11. Perez-Alvarez $R$, Perez-de-Lis $M$, Diaz-Lagares $C$, Pego-Reigosa JM Retamozo S, et al. (2011) Interstitial lung disease induced or exacerbated by TNF-targeted therapies: analysis of 122 cases. Semin Arthritis Rheum 41 : 256-264.

12. Garg K, Lynch DA, Newell JD (1993) Inflammatory airways disease in ulcerative colitis: CT and high-resolution CT features. J Thorac Imaging 8: 159-163.

13. Mahadeva R, Walsh G, Flower CD, Shneerson JM (2000) Clinical and radiological characteristics of lung disease in inflammatory bowel disease. Eur Respir J 15: 41-48.

14. Papanikolaou I, Kagouridis K, Papiris SA (2014) Patterns of airway involvemen in inflammatory bowel diseases. World J Gastrointest Pathophysiol 5: 560-569.

15. Godet PG, Cowie R, Woodman RC, Sutherland LR (1997) Pulmonary function abnormalities in patients with ulcerative colitis. Am J Gastroenterol 92: 1154-1156.

16. Mohamed-Hussein AA, Mohamed NA, Ibrahim ME (2007) Changes in pulmonary function in patients with ulcerative colitis. Respir Med 101: 977-982.

17. Storch I, Sachar D, Katz S (2003) Pulmonary manifestations of inflammatory bowel disease. Inflamm Bowel Dis 9: 104-115. 